\title{
Mechanism of transmembrane and coiled-coil domain 1 in the regulation of proliferation and migration of A549 cells
}

\author{
CHEN YANG* ${ }^{*}$ YUAN WANG*, JIAN-QI BAI, JING-RU ZHANG, PEI-YAN HU, \\ YAN ZHU, QIN OUYANG, HONG-MEI SU, QIU-YUE LI and PING ZHANG
}

Department of Pathology, Wangjing Hospital, China Academy of Chinese Medical Sciences, Beijing 100102, P.R. China

Received August 26, 2019; Accepted June 29, 2020

DOI: $10.3892 / \mathrm{ol} .2020 .12020$

\begin{abstract}
Bioinformatics analyses have shown that transmembrane and coiled-coil domain 1 (TMCO1) may be associated with lung adenocarcinoma. However, to the best of our knowledge, no current research has determined whether TMCO1 is involved in the development of lung adenocarcinoma. The present study aimed to identify the association between TMCO1 and lung adenocarcinoma. The present study demonstrated that the positive immunohistochemical staining of TMCO1 in lung adenocarcinoma tissues was significantly higher compared with paracarcinoma tissues. Additionally, knockdown of TMCO1 was demonstrated to downregulate B-cell lymphoma-2 protein expression levels and upregulate cysteinyl aspartate specific proteinase (caspase)-3 and caspase-9 protein expression levels in A549 cells. These changes resulted in decreased apoptosis of A549 cells uponTMCO1 downregulation. In addition, knockdown of TMCO1 decreased matrix metalloproteinase (MMP)-2 and MMP-9 expression levels. The expression of $\mathrm{N}$-cadherin and vimentin also decreased. By contrast, the expression levels of E-cadherin protein increased. Knockdown of TMCO1 resulted in the inhibition of A549 cell migration. The results of the present study demonstrated that TMCO1 was associated with lung adenocarcinoma and that inhibition of TMCO1 expression levels negatively regulated the apoptosis and migration of lung adenocarcinoma cells. Therefore, the present study suggests the potential for TMCO1 to be used in the clinical treatment of lung adenocarcinoma.
\end{abstract}

Correspondence to: Dr Ping Zhang, Department of Pathology, Wangjing Hospital, China Academy of Chinese Medical Sciences, 6 Huajiadi Street, Chaoyang, Beijing 100102, P.R. China

E-mail: wjpathologylab@sina.com

*Contributed equally

Abbreviation: TMCO, transmembrane and coiled-coil domain

Key words: lung adenocarcinoma, A549, proliferation, migration

\section{Introduction}

Calcium $\left(\mathrm{Ca}^{2+}\right)$ is sensitive to external stimuli and participates in cellular metabolic activities. Therefore, maintaining homeostasis of $\mathrm{Ca}^{2+}$ is crucial for maintaining normal cell structure and cellular functions. The intracellular calcium concentration is strictly and precisely controlled. This specific control is critical for controlling proteins and signaling pathways mediated by $\mathrm{Ca}^{2+}$ in the regulation of cell proliferation, apoptosis, gene transcription, and migration $(1,2)$. Abnormal intracellular $\mathrm{Ca}^{2+}$ concentrations cause a number of metabolic dysfunctions. The disorder of intracellular calcium affects the biological behaviors of tumor cells, such as proliferation and migration. A number of studies have shown that calcium-mediated signaling pathways are implicated in the occurrence and development of tumors $(3,4)$.

A previous study demonstrated that transmembrane and coiled-coil domain 1 (TMCO1), an endoplasmic reticulum transmembrane protein, can actively regulate intracellular calcium concentrations (5). The present study confirmed that TMCO1 can recognize intracellular $\mathrm{Ca}^{2+}$ concentration and form calcium channels, actively discharging intracellular overloaded $\mathrm{Ca}^{2+}$, providing functions that are crucial for cellular calcium homeostasis. Studies have demonstrated that TMCO1 is associated with skeletal development and glaucoma $(6,7)$. Li et al (8) revealed that TMCO1 participated in the regulation of proliferation and migration of bladder urothelial carcinoma via the AKT pathway, which demonstrated that TMCO1 is involved in the development of tumors.

Lung cancer has the highest incidence of cancer morbidity and mortality worldwide (9). Non-small cell carcinoma accounts for $84 \%$ of lung cancers and the major phenotype is lung adenocarcinoma (10). The specific pathogenesis and mechanism of lung adenocarcinoma development are currently unknown. However, evidence indicated that $\mathrm{Ca}^{2+}$ channels play an important role in proliferation and migration of lung adenocarcinoma cells (11).

Bioinformatic analysis demonstrated that there may be an association between TMCO1 and lung adenocarcinoma. However, the molecular mechanism involved in the association between TMCO1 and lung adenocarcinoma has not yet been determined. Thus, the present study aimed to investigate the underlying molecular mechanism of TMCO1 in regulating the biological process of A549 cells. 


\section{Materials and methods}

Patient and tissue samples. A total of seven patients with lung adenocarcinoma, including three males (age, $41-59$ years) and four females (age, 38-62 years), whose disease was pathologically confirmed at the China Academy of Chinese Medical Sciences of Wangjing Hospital (Beijing, China) were selected. Tissue samples were collected between January 2019 and October 2019 and written informed consent was provided by all patients. The study was approved by the Ethics Committee of Wangjing Hospital (Beijing, China) and all patients agreed to participate.

Antibodies. TMCO1 antibody (rabbit) was obtained from Cleveland State University, Department of Chemistry and the Center for Gene Regulation in Health and Disease (Cleveland, OH, USA). $\beta$-actin antibody (rat; catalog no. CW0096) was purchased from CoWin Biosciences (CWBio). Bcl-2 (rabbit; catalog no. BA0412), caspase-3 (rabbit; catalog no. PB0183), caspase-9 (rabbit; catalog no. BA0690), MMP-2 (rabbit; catalog no. A00286), MMP-9 (rabbit; catalog no. BA0573), $\mathrm{N}$-cadherin (mouse; catalog no. BM1573), E-cadherin (rabbit; catalog no. BA0475), vimentin (rabbit; catalog no.PB9359) and calcium/calmodulin-dependent protein kinase II inhibitor 1 (CAMKII) antibodies (mouse; catalog no. M03241-1) were purchased from Boster Biological Technology.

Bioinformatics analysis. GeneExpression Profiling Interactive Analysis (GEPIA; http://gepia.cancer-pku.cn/) is an online tool that provides expression level analysis functions for The Cancer Genome Atlas (TCGA; https://www.cancer. gov/about-nci/organization/ccg/research/structural-genomics/tcga) and the Genotype-Tissue Expression (https://commonfund. nih.gov/GTEx) databases. In the present study, GEPIA was used to compare the expression levels of TMCO1 in lung adenocarcinoma ( $\mathrm{T}=483)$ which all come from TCGA tumor data and normal lung tissues $(n=347)$, which were matched TCGA normal and GTEx data, the distinct expression of different stages of lung adenocarcinoma tissues in Tumor Node Metastasis (TNM) standard, as well as the overall survival and progression-free survival of the patients. In the expression level analysis of TMCO1 for the different sub-stages, $\log 2$ transcripts per million $(\mathrm{TPM}+1)$ of the RNA sequencing expression level data was used for the log-scale. One-way ANOVA was performed, using the pathological stage as a variable to calculate the differential expression. In the survival analysis, GEPIA online tools used the log-rank test (also known as the Mantel-Cox test) for the hypothesis test. The Cox proportional hazard ratio and the $95 \%$ confidence interval information were also included in the survival plots. To investigate survival analysis of TMCO1, patients with lung adenocarcinoma were classified into the high expression cohort and low expression cohort groups, according to the median expression (the median expression threshold was 50\%).

Immunohistochemical staining. The tissues (including the lung adenocarcinoma tissues and the para-carcinoma tissues) were fixed in 10\% formalin solution (Beijing YiliFine Chemicals Co., Ltd) for $24 \mathrm{~h}$ at room temperature, then they were treated with Fully-enclosed tissue processor
(ASP300s, Leica), embedded in paraffinand sectioned. After that, the sections $(3 \mu \mathrm{m})$ was dewaxing in a dewaxing liquid (catalog no. BLB-01, Jiu Zhou Bailin Co., Ltd) and rehydrated in a graded ethanol seriesat room temperature for $15 \mathrm{~min}$ each stage. Tissue sections were blocked with $10 \% \mathrm{H}_{2} \mathrm{O}_{2}(20 \mathrm{ml}$ $\mathrm{H}_{2} \mathrm{O}_{2}$ dissolved in $180 \mathrm{ml}$ methyl alcohol, Beijing Chemical Works) at room temperature for $10 \mathrm{~min}$, followed by treatment with 3\% BSA (Beijing Solarbio Science \& Technology Co., Ltd.) at room temperature for $30 \mathrm{~min}$. The sections were incubated with rabbit polyclonal antibody TMCO1 (1:50) diluted in $2 \% \mathrm{BSA} / 0.1 \mathrm{M}$ PBS overnight at $4^{\circ} \mathrm{C}$ and further incubated with goat anti-rabbit immunoglobulin G (1:100) (IgG; catalog no. CW0103, CWBio) at room temperature for $1 \mathrm{~h}$. The sections were transferred to an avidin-biotin-peroxidase complex solution for $30 \mathrm{~min}$, then submerged in 3 , 3-diaminobenzidine (DAB; catalog no. CW0125; CWBio) for $1 \mathrm{~min}$, which produced brown staining. Hematoxylin (Beijing Solarbio Science \& Technology Co., Ltd.) was used for nuclear staining at room temperature. After $1 \mathrm{~min}$, the reaction was stopped with 0.1 M PBS and water rinsing. The slides were fully rinsed with $0.1 \mathrm{M}$ PBS between the individual steps. The tissue sections were rinsed in water, $70 \%$ ethanol, $95 \%$ ethanol, 100\% ethanol and xylenes. Subsequently, images were captured using a light microscope (magnification, $\mathrm{x} 400$; TE2000-s, Nikon) using Image Scope software (Nis-Elements D 2.30 software, Nikon). The number and density of positive-stained cells were analyzed by Image-Pro Plus 6.0 (Media Cybernetics) and GraphPad Prism 8.0 software (GraphPad Software, Inc.).

Cell culture and treatment. A549 cells were provided by Cleveland State University, Department of Chemistry and the Center for Gene Regulation in Health and Disease (Cleveland, USA), which purchased the cells from American Type Culture Collection (ATCC). The cells were divided into three groups: Untreated A549 cells served as the control group, while the 29 and 33 sites of TMCO1 were knocked down to create the A549-29- and A549-33- knockdown groups, respectively. The 29 and 33 sites were the main functional sites of TMCO1 as previously described (5) The cells were cultured in RPMI-1640 supplemented with 10\% fetal bovine serum (both from Beijing Solarbio Science \& Technology Co., Ltd.) and cultured at $37^{\circ} \mathrm{C}, 5 \% \mathrm{CO}_{2}$ in a humidified incubator (Heraeus Holding $\mathrm{GmbH}$ ).

Western blot analysis. The cells were lysed with radioimmunoprecipitation assay (RIPA lysis buffer) (Beyotime Institute of Biotechnology) and protein concentration was quantified using the bicinchoninic acid (CWBio) method. The proteins (20 $\mu \mathrm{g} / \mathrm{lane}$ ) were separated on 10\% SDS-PAGE (CWBio) and transferred onto PVDF membranes (CWBio). The membranes were blocked in 5\% milk (Inner Mongolia Yili Industrial Group Co., Ltd)-TBS-Tween-20 (CWbio) for $1 \mathrm{~h}$ at room temperature. Then, the membranes were incubated with primary antibodies ( $\beta$-actin, 1:1,000 andTMCO1; 1:500) overnight at $4^{\circ} \mathrm{C}$, then incubated with a secondary antibody (goat anti-rabbit IgG-horseradish peroxidase; 1:3,000, catalog no. CW0103, $\mathrm{CWBio}$ ) for $2 \mathrm{~h}$ at room temperature. Finally, the immunoreactive bands were detected using the EasySee Western Blot kit (CWBio). 
Detection of calcium ion concentration. A549 cells were seeded $\left(1 \times 10^{5}\right)$ in a $20-\mathrm{mm}$ culture dish. After $24 \mathrm{~h}, 4 \mu \mathrm{m} / \mathrm{ml}$ of Fulo-3 solution (50 $\mu \mathrm{g}$ Fulo-3 dissolved in $44 \mu \mathrm{l}$ DMSO, diluted with $11 \mathrm{ml}$ PBS; Thermo Fisher Scientific, Inc.) was added to the cells and incubated for $40 \mathrm{~min}$ at $37^{\circ} \mathrm{C}$. The cells were washed twice with PBS followed by addition of RPMI-1640 medium (Beijing Solarbio Science \& Technology Co., Ltd.) for 30 min. Subsequently, Hank's Balanced Salt Solution (HBSS; Beijing Solarbio Science \& Technology Co., Ltd.) containing $\mathrm{Ca}^{2+}$ was added and the cells were scanned under a confocal microscope (Olympus Corporation, magnification, x400) for $300 \mathrm{sec}$. After $60 \mathrm{sec}$ of scanning, $100 \mu \mathrm{l}$ ATP (Beijing Solarbio Science \& Technology Co., Ltd.) solution $(300 \mathrm{~m} / \mathrm{ml}$, $0.125 \mathrm{~g}$ ATP2Na dissolved in $4 \mathrm{ml}$ HBSS) was added at room temperature. The images and ratio images were captured using Olympus Fluoview 3.1a software (Olympus Corporation). Monitoring of $\left[\mathrm{Ca}^{2+}\right]$ required two continuous images ( $\mathrm{F} 1$ and $\mathrm{F} 2)$ the ratio images $\mathrm{R}=\mathrm{F} 1 / \mathrm{F} 2$ were used to derive $\left[\mathrm{Ca}^{2+}\right]$. Data analysis was conducted in Microsoft Excel 2010 (Microsoft Corporation).

Cell activity analysis. A549 cells (1×10/well) were seeded into a 96-well plate. MTT solution (Beijing Solarbio Science $\&$ Technology Co., Ltd.) was added following $48 \mathrm{~h}$ of culture and the cells were incubated for $4 \mathrm{~h}$ at $37^{\circ} \mathrm{C}$. Subsequently, $50 \mu \mathrm{l}$ DMSO was added to each well and the absorbance was measured at a wavelength of $490 \mathrm{~nm}$ using a microplate reader. Data analysis was performed using GraphPad Prism 8.0 software (GraphPad Software, Inc.).

Cell migration assay by scratch test and Transwell. A549 cells $\left(1 \times 10^{5} /\right.$ well) were seeded into a 24 -well plate and the experiment proceeded when cells reached $100 \%$ confluency. A $200-\mu 1$ pipette tip was used to make a scratch wound in the cells, which were then cultivated in 1640 medium without serum. Cell migration pictures were captured under a light microscope (magnification, $\mathrm{x} 40$ ) using Nis-Elements D 2.30 software (Nikon) at $0,6,12$ and $24 \mathrm{~h}$ after the scratch wound was made. The migration rate was analyzed by ImageJ (National Institutes of Health) and GraphPad Prism 8.0 (GraphPad Software, Inc.) software.

Cell suspension $\left(1 \times 10^{4} /\right.$ well, $\left.100 \mu \mathrm{l}\right)$ was added into the upper chambers (Corning, Inc.). Medium containing 10\% fetal bovine serum (Solarbio) was added to the lower chamber of the 24-well plate and the cells were incubated for $24 \mathrm{~h}$ at $37^{\circ} \mathrm{C}$. Subsequently, cells were fixed with $90 \%$ alcohol for $15 \mathrm{~min}$ at room temperature and cell migration was observed in a light microscope. Images were captured (magnification, x20) following Coomassie blue staining for $1 \mathrm{~min}$ at room temperature using Nis-Elements D 2.30software. The number and density of positive stained cells were analyzed by Image Pro Plus 6.0and GraphPad Prism 8.0 (GraphPad Software, Inc.) software.

Immunocytochemical staining. A549 cells (1x105/well) were seeded in a 24 -well plate and fixed at $4^{\circ} \mathrm{C}$ with $10 \%$ formaldehyde following $24 \mathrm{~h}$ incubation. Subsequently, cells were washed twice with PBS and incubated with $10 \% \mathrm{H}_{2} \mathrm{O}_{2}\left(20 \mathrm{ml} \mathrm{H}_{2} \mathrm{O}_{2}\right.$ dissolved in $180 \mathrm{ml}$ methyl alcohol, Beijing Chemical Works) for $10 \mathrm{~min}$ at room temperature. Cells were then incubated for $30 \mathrm{~min}$ at room temperature with 5\% BSA for blocking and antibodies (Bcl2, 1:50; caspase-3, 1:100; caspase-9, 1:100; MMP-2, 1:50; MMP-9, 1:50; N-cadherin, 1:50; E-cadherin, 1:50; vimentin, 1:100 and CAMKII, 1:50) were added for $1 \mathrm{~h}$ at $37^{\circ} \mathrm{C}$. After rinsing twice with PBS, secondary antibody (1:100; goat anti-rabbit IgG, catalog no. CW0103; CWBio; goat Anti-Mouse IgG, catalog no. CW0102, CWBio) was added for $30 \mathrm{~min}$ at room temperature and the cells were observed following DAB chromophore staining for $1 \mathrm{~min}$ at room temperature and images were obtained in a light microscope. The integral optical density values of the images (magnification, x100) were analyzed using Image-Pro Plus 6.0 (Media Cybernetics) and GraphPad Prism 8.0 (GraphPad Software, Inc.) software.

Statistical analysis. Survival analysis was assessed using the log-rank test. The bioinformatic expression analysis of TMCO1 used one-way ANOVA. Cell culture experiments were repeated at least three times. Unpaired independent samples t-test or one-way ANOVA was used for comparisons between groups. Multiple group comparisons of Bcl-2 were analyzed by LSD and the Caspase- 3 and Caspase- 9 expression were analyzed by the Duncan's test as a post-hoc test. Bonferroni's was also used following one-way ANOVA as a post-hoc test for cell migration, MMPs and EMT factors analyzed. All statistical analysis was performed using SPSS for Windows (version 18.0; SPSS, Inc.). $\mathrm{P}<0.05$ was considered to indicate a statistically significant difference. Histograms were produced using GraphPad Prism 8.0 software (GraphPad Software, Inc.).

\section{Results}

TMCO1 is associated with lung adenocarcinoma development and high expression levels in lung adenocarcinoma tissue. The bioinformatics analysis results showed that TMCO1 expression levels were high in lung adenocarcinoma tissues compared with normal tissues (Fig. 1A). Additionally, TMCO1 expression levels in stage I of the disease was lower compared with other stages (Fig. 1B). Meanwhile, the expression levels of TMCO1 affected the survival rate; high expression levels of TMCO1 were associated with a significantly decreased survival rate (dotted lines represent 95\% confidence interval) (Fig. 1C). These results indicated a potential association between TMCO1 and biological functions of tumors. Positive staining of TMCO1 was observed in the lung adenocarcinoma tissues and paracarcinoma tissues of these patients. Positive TMCO1 staining in LUAD tissues was notably higher than in paracarcinoma tissues (Fig. 1D). The results demonstrated that TMCO1 was associated with lung adenocarcinoma. To clarify the association between TMCO1 and lung adenocarcinoma, A549 cells were selected for the subsequent experiments.

Increased intracellular calcium concentration following TMCO1 knockdown. The present study examined the expression levels of TMCO1 protein in each group of A549 cells using western blotting. The results demonstrated that the expression levels of TMCO1 in the A549-29- and A549-33- groups were notably decreased compared with the control group, indicating that TMCO1 expression levels were successfully 


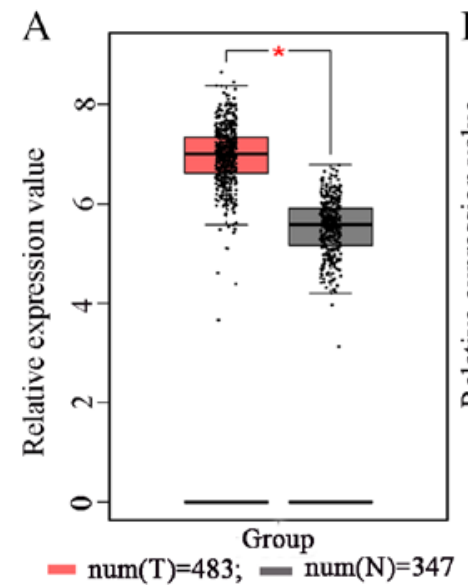

$\mathrm{C}$
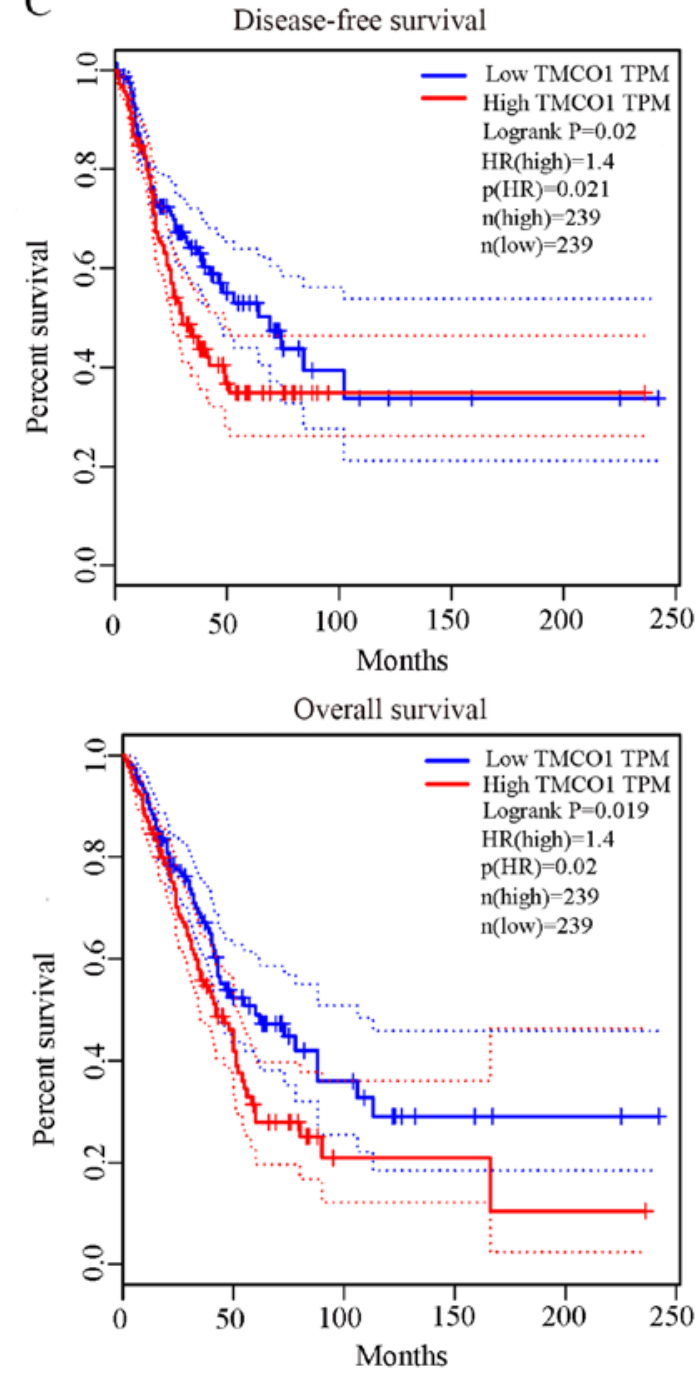

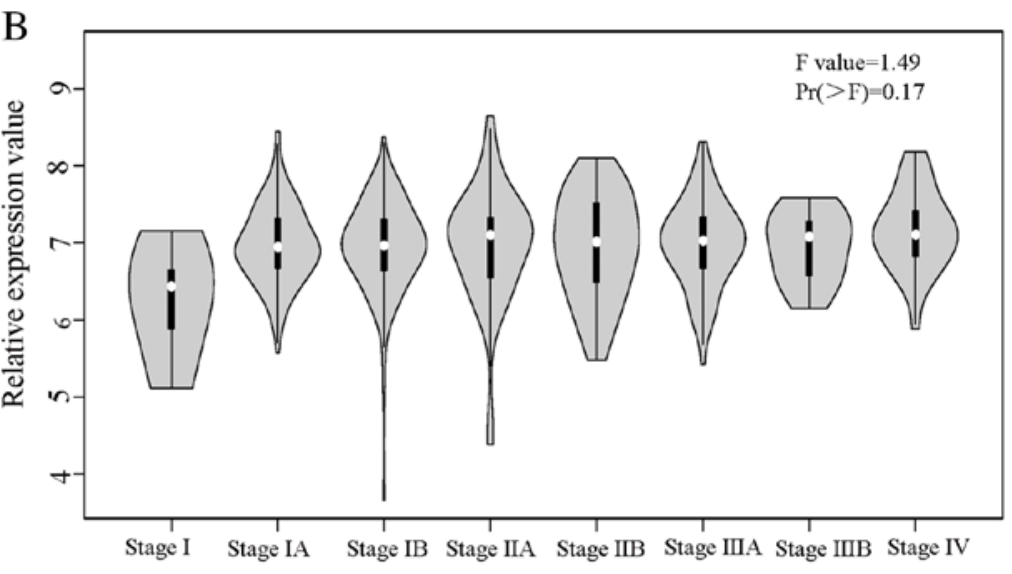

$\mathrm{D}$

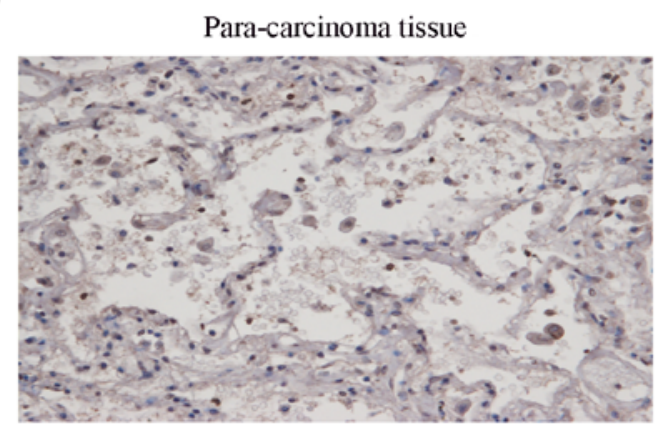

LUAD tissue

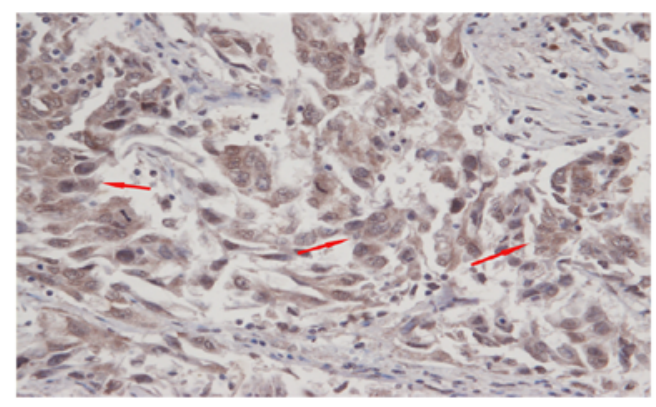

TMCOI expression

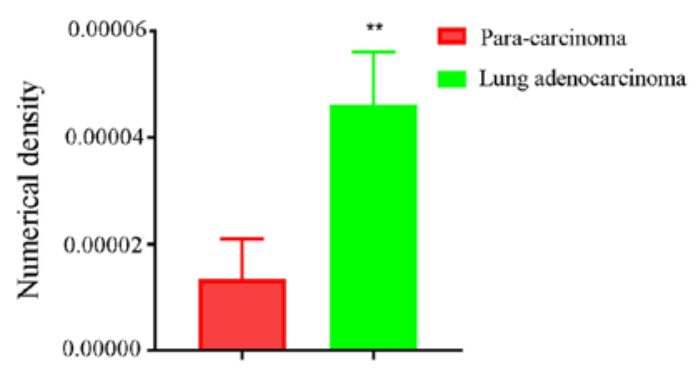

Figure 1. Association between TMCO1 and LUAD. (A) Biological analysis of expression levels of TMCO1 in patients with LUAD and the control group. (B) Bioinformatics analysis of expression levels of TMCO1 in different stages of LUAD. (C) Bioinformatics analysis showing the association between TMCO1 and survival rates (dotted lines represent 95\% confidence interval). (D) The results showed that positive TMCO1 staining in LUAD tissues was notably higher than in paracarcinoma tissues (red arrows; magnification, $\mathrm{x} 400$ ). Data are expressed as the mean \pm standard deviation. ${ }^{*} \mathrm{P}<0.05,{ }^{* *} \mathrm{P}<0.01 \mathrm{vs}$. control. TMCO, transmembrane coiled-coil domain; LUAD, lung adenocarcinoma; TPM, transcripts per million; HR, hazard ratio.

knocked down (Fig. 2A). CAMKII protein expression levels in the A549-29- and A549-33- group were slightly decreased compared with the control group (Fig. 2B), indicating that CAMKII expression was inhibited after TMCO1 knockdown. In addition, confocal microscopy revealed that the intracel- lular calcium concentration after TMCO1 knockdown in the A549-29- and A549-33- groups was higher compared with the control group (Fig. 2C). These assays demonstrated that knocking down TMCO1 could cause $\mathrm{Ca}^{2+}$ overload in A549 cells, decreasing CAMKII expression levels. 
A
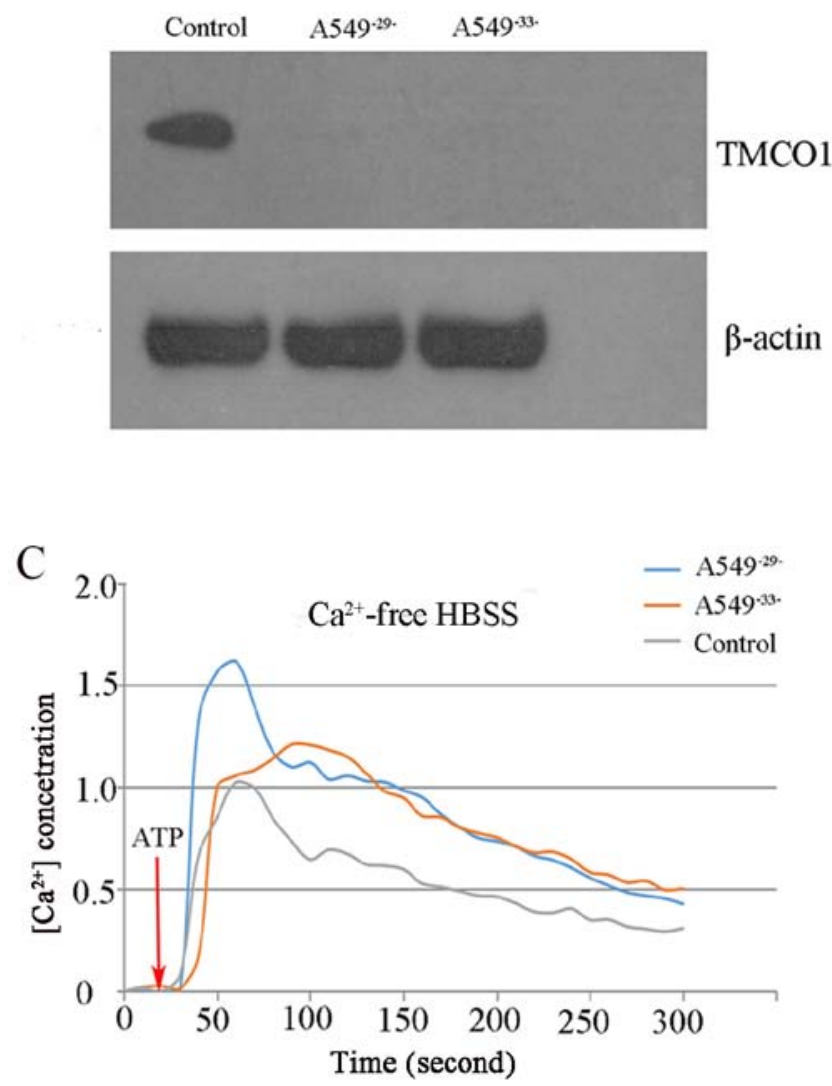

B
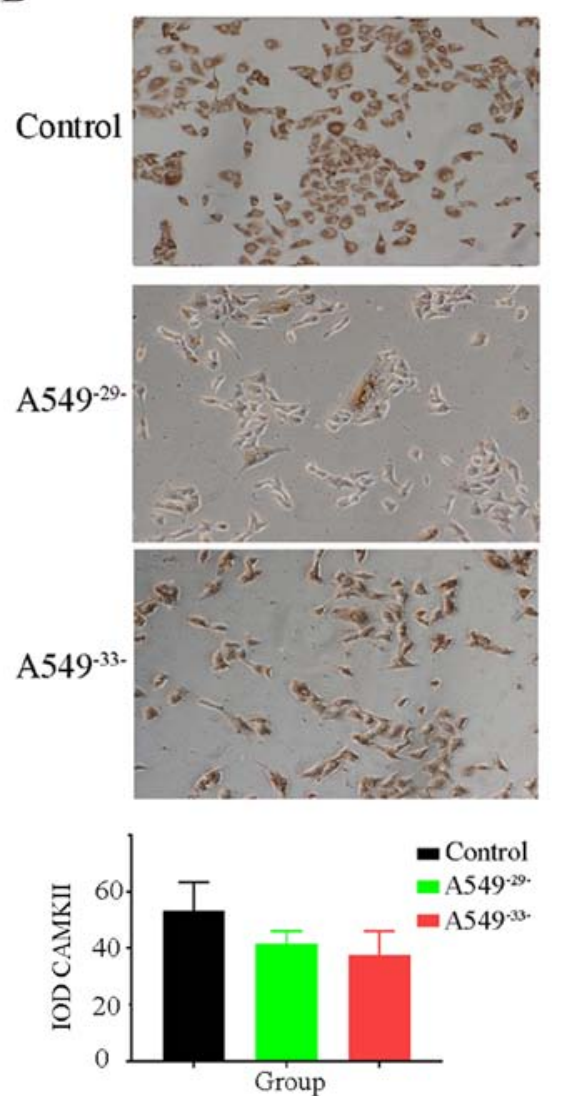

Figure 2. Changes in A549 cells following TMCO1 knockdown. (A) The results showed that the expression levels of TMCO1 in the A549-29- and A549-33groups were notably decreased compared with the control group. (B) The result showed that CAMKII protein expression in the A549-29- and A549-33- group were slightly decreased compared with the control group (magnification, x100). (C) Detection of calcium ion concentration result showed that the intracellular calcium concentration in A549-29- and A549-33- groups was higher compared with the control group. Monitoring of [Ca ${ }^{2+}$ ] required two continuous images (F1 and F2) the ratio images R=F 1/F 2 were used to derive [ $\left.\mathrm{Ca}^{2+}\right]$ TMCO1, transmembrane coiled-coil domain; HBSS, Hank's Balanced Salt Solution; IOD, integrated optical density; CAMKII, calcium/calmodulin-dependent protein kinase II inhibitor 1.

TMCO1 knockdown decreases cell activity and affects cell apoptosis. MTT results showed that the activity of the A549-29-knockdown cells was three-fold lower compared with control group, and the cell activity of A549-33- group was also significantly decreased. The results indicated that A549 cell activity was significantly inhibited by TMCO1 knockdown (Fig. 3A). Meanwhile, Bcl-2 expression levels markedly decreased in the A549-29- group compare to the control. By contrast, the expression levels of caspase-3 were significantly increased in the A549-33- group compared with the control group. Caspase-9 protein expression levels were significantly upregulated in the A549-29-group compared with the control group (Fig. 3B). The results suggest that TMCO1 regulated cell apoptosis by affecting Bcl-2, caspase- 3 and caspase- 9 expression levels.

Cell migration ability decreases following TMCO1 knockdown. Following the scratch test, the migration of the A549-29-cells and $A 549^{-33}$ cellsweresuppressed after $12 \mathrm{~h}$ compared with the control group. The migration rate of the A549-29- cells significantly decreased compared with the control group over time, indicating that TMCO1 exerted a certain regulatory effect on the A549 cell migration process (Fig. 4A). The Transwell assay demonstrated that the cell migration rate in the A549-29- group was significantly lower compared with the control group after $24 \mathrm{~h}$ and the migration of cells in the A549-33- group was also markedly lower compared with the control group (Fig. 4B). Examination of migration-associated factors showed that MMP-2 expression levels were significantly lower in the A549-33- group compared with controls, and MMP-9 protein expression levels in the A549-29- group significantly decreased compared with the control group (Fig. 4C). In addition, knockdown of TMCO1 downregulated N-cadherin and vimentin expression levels, which were decreased in the A549-33- group, whereas E-cadherin expression levels were significantly increased in the A549-33- group compared with the control group (Fig. 4D). The results showed that MMP-2 and MMP-9 expression levels were decreased by inhibiting TMCO1 expression levels, accompanied by decreases in $\mathrm{N}$-cadherin and vimentin expression levels and increases in E-cadherin expression levels, resulting in a significant decrease to the migration ability of the A549 cells.

\section{Discussion}

Proliferation and migration are the primary characteristics of cancer. Therefore, identification of their pathological mechanism is an important theoretical basis for clinical treatment. 
A

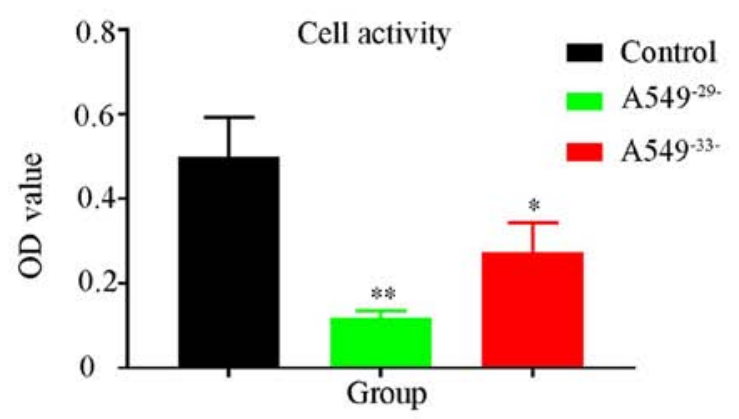

B
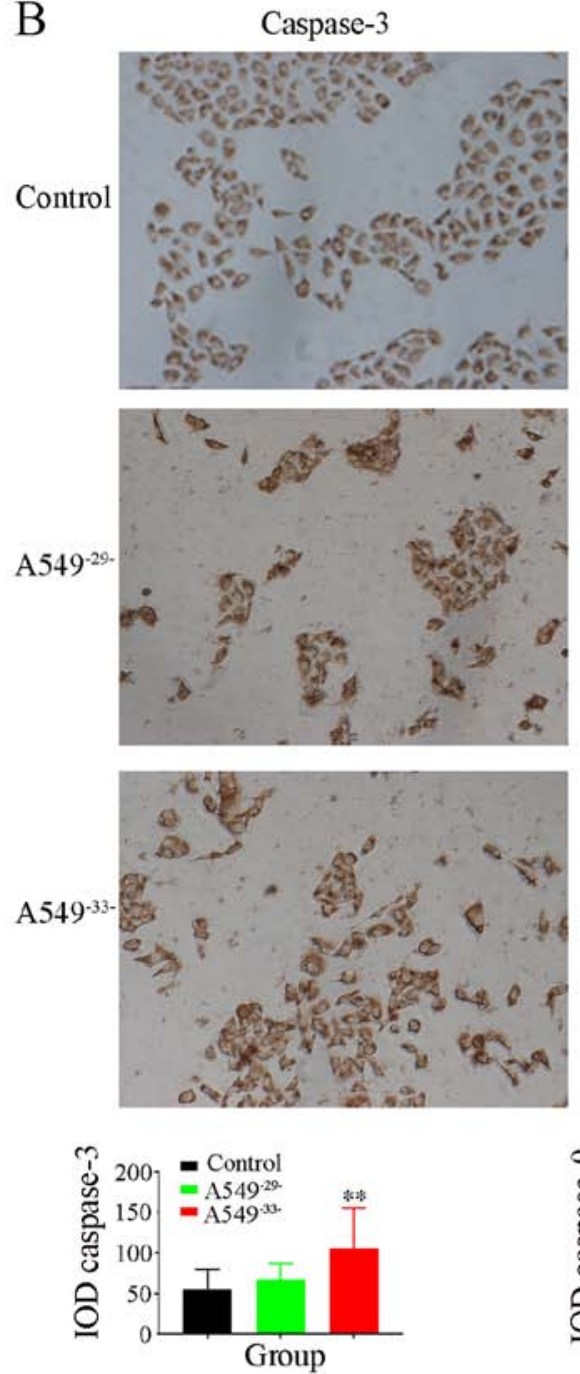
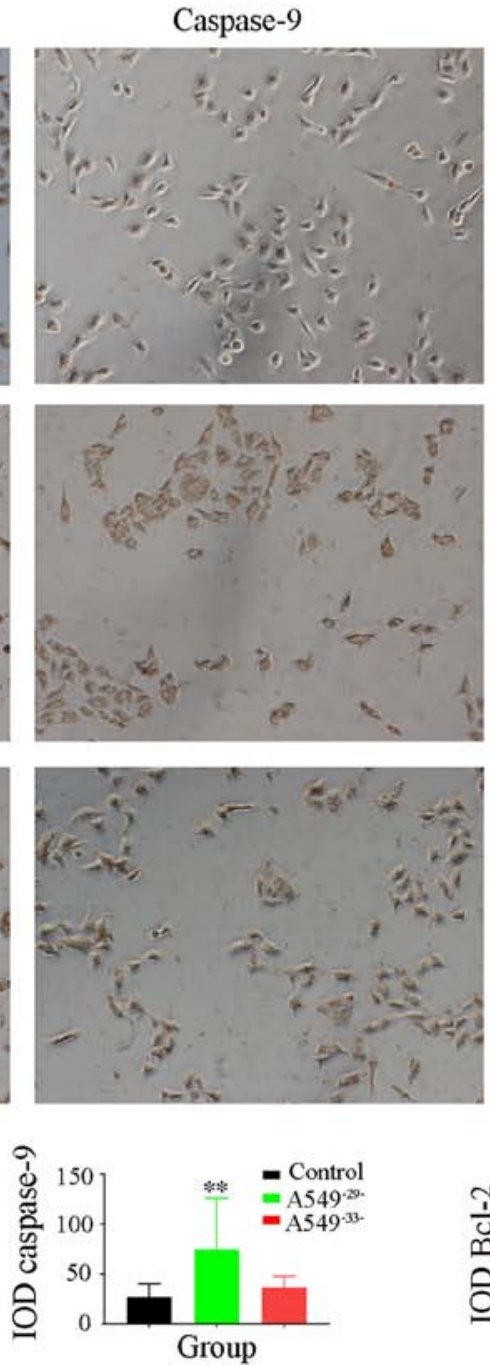

Bcl-2
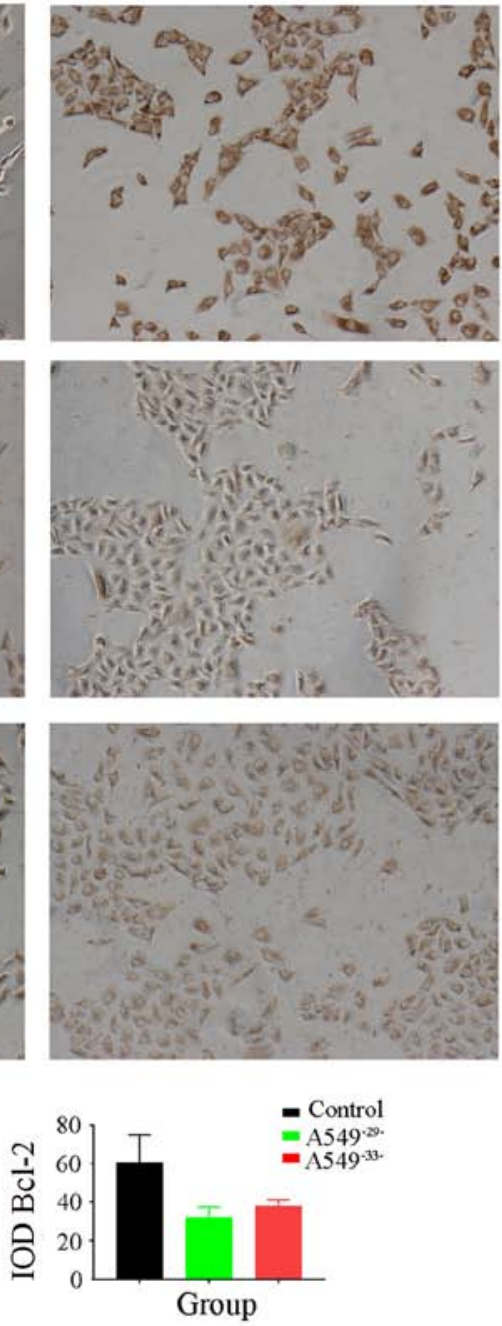

Figure 3. Detection of cell activity and the expression levels of Bcl-2, Caspase-3 and Caspase-9 in A549 cells. (A) MTT results showed that the activity of the A549-29-knockdown cells was three-fold lower compared with control group, and the cell activity of A549-33- group was also significantly decreased compared with control. (B) The Bcl-2 expression levels markedly significantly decreased in the A549-29- group and A549-33- group compare to the control. Caspase-3 expression significantly increased in the A549-33- group compared with the control group. Caspase-9 protein expression significantly increased in the A549-29-group compared with the control group (magnification, x100). Image Pro Plus software was used to analyze the images. Data are presented as the mean \pm standard deviation. ${ }^{*} \mathrm{P}<0.05,{ }^{* *} \mathrm{P}<0.01$ vs. control. OD, optical density; IOD, integrated optical density.

Lung adenocarcinoma is a multifactorial disease and its proliferation and migration processes involve numerous signaling pathways and factors, such as $\mathrm{Ca}^{2+}(12,13)$. Studies have shown that active calcium ions can stimulate tumor cell proliferation and trigger drug resistance $(14,15)$. Yang et al (16), demonstrated that decreasing intracellular calcium concentrations decreased the proliferation and migration of A549 cells. However, none of these studies demonstrated whether intracellular calcium overload affected the biological function of A549 cells. Moreover, to the best of our knowledge, no studies have ever clarified that TMCO1 was associated with lung adenocarcinoma biological processes. In the present study, bioinformatics analysis showed that TMCO1 expression levels in tissue from patients with lung adenocarcinoma were higher compared with normal tissue samples. The results of the present study also demonstrated that TMCO1 expression levels were inversely proportional to the survival rate. These results indicated that TMCO1 may be associated with lung adenocarcinoma. 
A
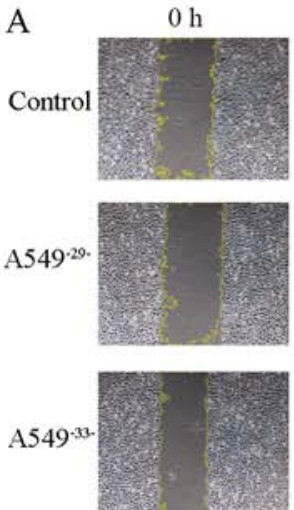

C
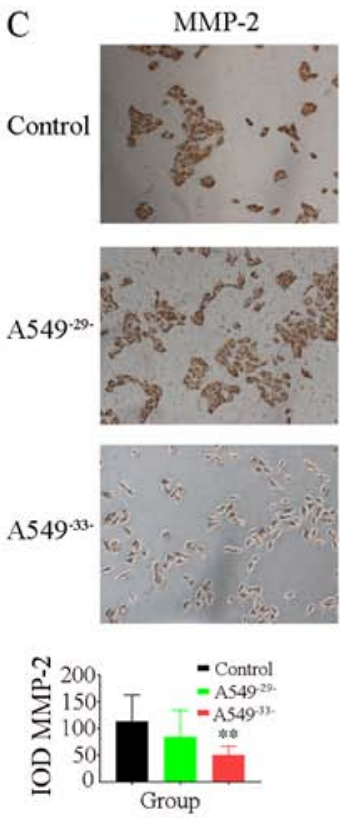

$6 \mathrm{~h}$
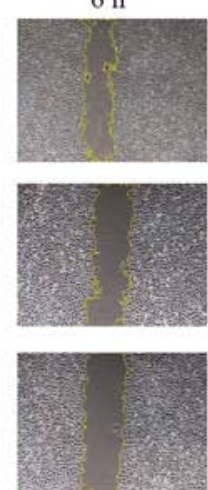

$12 \mathrm{~h}$
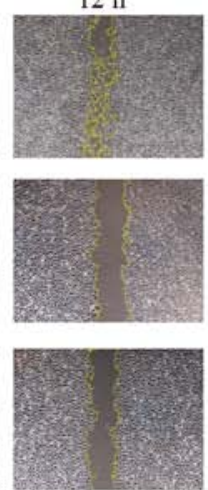
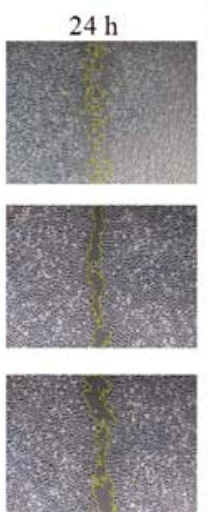

D N-cadherin
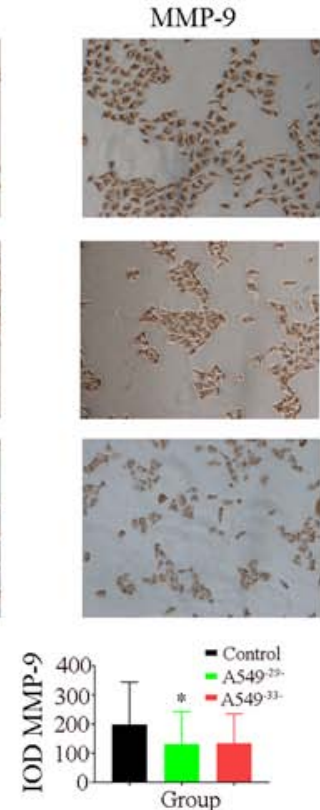
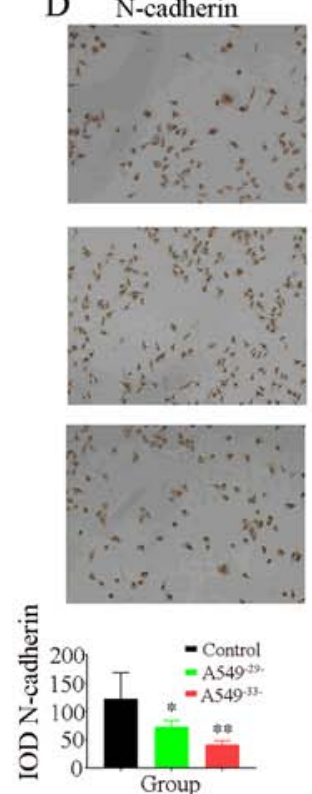

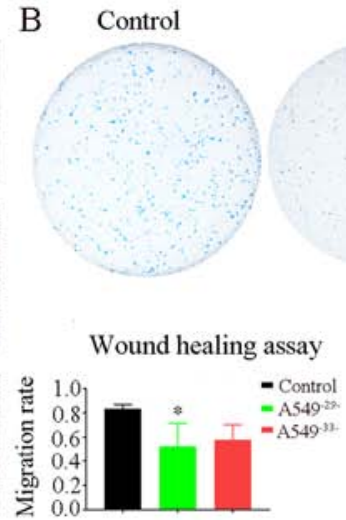

E-cadherin
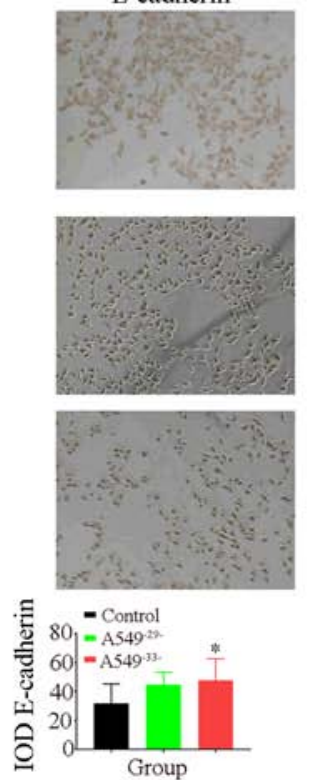

A549-29.
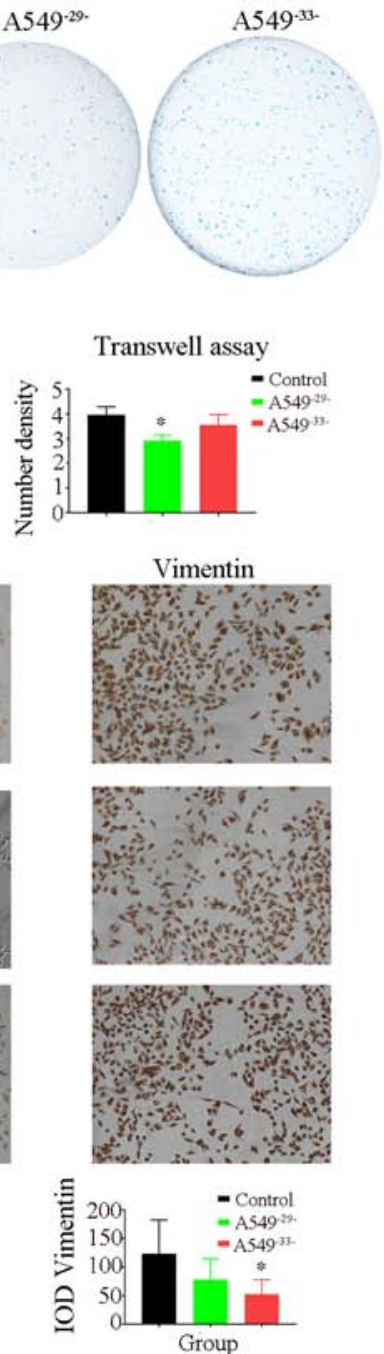

Figure 4. Detection of migration activity and expression levels of MMP-2, MMP-9, N-cadherin, E-cadherin and Vimentin in A549 cells. (A) Scratch test showed that the migration of A549-29-and A549-33-cells were suppressed after $12 \mathrm{~h}$ compared with the control group. The migration rate of the A549-29- cells significantly decreased compared with the control group over time. The migratory rate slightly decreased in the A549-33- group compared with the control group (magnification, $\mathrm{x} 40$ ). (B) The Transwell results showed that the cell migration rate in the A549-29- group was significantly lower compared with the control group after $24 \mathrm{~h}$ and the migration of cells in the A549-33- group was also lower compared with the control group (magnification, $\mathrm{x} 20$ ). (C) The results

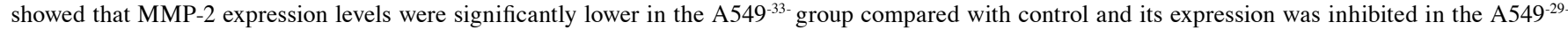
group compare with the control. The MMP-9 protein expression levels in the A549-29- group significantly decreased compared with the control group and its expression in A549-33- was also less than the control (magnification, 100X). (D) The results showed that the expressions of N-cadherin and Vimentin in the A 549-29- and A549-33- groups were lower than the control group. In addition, the expression of E-cadherin in the A549-29- and A549 ${ }^{-33-}$ groups was higher than the control group (magnification, x100). Image Pro Plus software was used to analyze the images. Data are expressed as the mean \pm standard deviation. "P<0.05, ${ }^{* *} \mathrm{P}<0.01$ vs. control. MMP, matrix metalloproteinase; IOD, integrated optical density.

Previous studies have demonstrated that $\mathrm{Ca}^{2+}$ are active in cancer cells at abnormally elevated concentrations and stimulate the proliferation and migration of cancer cells $(17,18)$. Using immunohistochemical staining, the present study demonstrated that the positive staining of TMCO1 expression levels was higher in lung adenocarcinoma tissues compared with paracarcinoma tissues. When the concentration of calcium ions in the lung adenocarcinoma cells is high, it may activate TMCO1 to regulate intracellular calcium.

Classical lung adenocarcinoma A549 cells were used to demonstrate that knockdown of the 29 or 33 sites of TMCO1 triggered intracellular calcium overload, consistent with the research results of Wang et al (5), demonstrating that TMCO1 can prevent intracellular calcium overload. As a mediator of $\mathrm{Ca}^{2+}$ signaling, CAMKII can affect numerous biological cell functions, including cell proliferation and migration, by regulating multiple steps of the calcium cycle (19). The present study demonstrated that inhibiting TMCO1 expression levels in A549 cells could cause $\mathrm{Ca}^{2+}$ overload, leading to decreased CAMKII expression levels. The present study demonstrated that TMCO1 expression levels are active in lung adenocarcinoma tissues and participate in regulating calcium concentration in A549 cells, indicating that there is an association between TMCO1 and lung adenocarcinoma.

In order to determine the regulatory mechanism of TMCO1 in the A549 cell activity, the present study examined A549 cell proliferation using MTT. The results showed that the activity of A549 cells decreased significantly after inhibiting TMCO1 expression levels, suggesting that TMCO1 knockdown could slow down A549 cell prolif- 
eration. Byun et al (20) also found that A549 cell activity was decreased by KCP10043F treatment to inhibit $\mathrm{Ca}^{2+}$ expression levels, which was consistent with the results of the present study. Expression levels of apoptotic proteins were measured, which demonstrated that the knockdown of TMCO1 inhibited the expression level of Bcl-2. Previous studies showed that Bcl-2 and A549 cell proliferation were inversely associated and $\mathrm{Ca}^{2+}$ was closely associated with Bcl-2 through multiple targets and pathways in cell proliferation and apoptotic processes $(21,22)$. The present study demonstrated that, following the knockdown of TMCO1 in A549 cells, intracellular calcium overload was provoked, resulting in a decrease in $\mathrm{Bcl}-2$ protein expression levels. In addition, as a second messenger in cells, calcium ions are associated with other factors, such as caspases (23) involved in cell apoptosis. In order to investigate this, the present study examined the expression levels of caspase-3 and caspase-9. Li et al (24) demonstrated that the apoptosis regulators caspase- 3 and caspase- 9 are active when A549 cell proliferation was decreased by angelicin treatment. Similarly, the present study demonstrated that the expression levels of apoptotic factors caspase- 3 and caspase- 9 were higher following TMCO1 knockdown compared with the control group. Therefore, the present study indicated that TMCO1 plays a central role in regulating the apoptosis of A549 cells. This function may be achieved by downregulating Bcl-2 expression levels and upregulating caspase-3 and caspase-9 protein expression levels in A549 cells.

Using scratch and Transwell experiments, the present study also demonstrated that knockdown of TMCO1 inhibited the migration of A549 cells. The migration ability of the knockdown group cells exhibited different degrees of attenuation compared with the control group. Further tests showed that knockdown of TMCO1 could downregulate the expression levels of MMP-2 and MMP-9. The MMP family is one type of calcium-dependent proteolytic enzyme that is directly involved in lung adenocarcinoma migration (25). Shi et al (26), showed that decreasing the expression levels of MMP-2 and MMP-9 could hinder lung adenocarcinoma cell migration, consistent with the results of the present study. Furthermore, epithelial-to-mesenchymal transition (EMT) has a critical role in tumor metastasis (27). As EMT markers, the downregulation of adhesion factor E-cadherin and the upregulation of N-cadherin expression levels are accompanied by migration and invasion in lung adenocarcinoma cells $(28,29)$. The present study demonstrated that TMCO1 knockdown could downregulate the expression levels of $\mathrm{N}$-cadherin and vimentin and upregulate the expression level of E-cadherin, indicating that the knockdown of TMCO1 inhibited the EMT process in A549 cells. As an important marker of EMT, vimentin expression levels increase significantly during tumor migration (30). The results of the present study indicated that TMCO1 can participate in regulating A549 cell migration by mediating MMP-2, MMP-9 and EMT activity.

In conclusion, the present study demonstrated that TMCO1 was closely associated with the cell activity, apoptosis and migration of lung adenocarcinoma cells. TMCO1 affected the apoptosis of lung adenocarcinoma cells by regulating the expression levels of $\mathrm{Bcl}-2$, caspase- 3 and caspase-9. Furthermore, it also regulated the expression levels of MMP-2,
MMP-9 and EMT processes to influence the migration of lung adenocarcinoma cells. The results demonstrated that TMCO1 had a relationship with A549 cells viability and migration. TMCO1, as a regulator of cellular calcium ions, was shown to affect numerous growth factors, which was in accordance with previous reports demonstrating that calcium ions participate in the regulation of numerous biological functions through complex networks $(31,32)$. In the present study, immunostaining was performed to observe changes in the expression levels of associated proteins, which suggested that TMCO1 participated in A549 cell biological processes. Future studies will verify changes in the expression levels of relevant factors at the gene level and determine the regulatory mechanism and regulatory targets of TMCO1 in lung adenocarcinoma to construct a complete network of TMCO1 regulatory mechanisms. To the best of our knowledge, the present study was the first to demonstrate an association between the proliferation, apoptosis and migration processes of lung adenocarcinoma, and TMCO1, which provides a new theoretical basis and potential clinical target for lung adenocarcinoma treatment and prognosis.

\section{Acknowledgements}

The authors would like to thank Dr Aimin Zhou (Cleveland State University, Department of Chemistry and the Center for Gene Regulation in Health and Disease, Cleveland, OH, USA) for providing the A549 cells.

\section{Funding}

The present study was funded by international cooperation projects of China Academy of Chinese Medical Sciences (grant no. GH2017-04-04) and TCM Clinical Base project of State Administration of Traditional Chinese Medicine (grant no. JDZX2015275).

\section{Availability of data and materials}

The datasets used and/or analyzed in the study are available from the corresponding author upon reasonable request.

\section{Authors' contributions}

PZ designed the study. CY and JQB performed western blot experiments. PYH collected the patient tissue samples. JRZ and QO performed and analyzed the IHC results. YW performed the cell culture and MTT experiments. HMS and QYL performed the Transwell assays. YZ collected and analyzed all the data. All authors participated in writing the manuscript and approved the manuscript. All authors read and approved the final manuscript.

\section{Ethics approval and consent to participate}

The present study was performed in accordance with The Code of Ethics of the World Medical Association. All patients agreed to participate in the present study and the study was approved by the Ethics Committee of Wangjing Hospital (Beijing, China; approval no. WJEC-KT-2019-019). 


\section{Patient consent for publication}

Not applicable.

\section{Competing interests}

The authors declare that they have no competing interests.

\section{References}

1. Moe AM, Golding AE and Bement WM: Cell healing: Calcium, repair and regeneration. Semin Cell Dev Biol 45: 18-23, 2015

2. Cui C, Merritt R, Fu L and Pan Z: Targeting calcium signaling in cancer therapy. Acta Pharm Sin B 7: 1-17, 2017.

3. Tennakoon S, Aggarwal A and Kállay E: The calcium-sensing receptor and the hallmarks of cancer. Biochim Biophys Acta 1863: 1398-1407, 2016.

4. Bong AHL and Monteith GR: Calcium signaling and the therapeutic targeting of cancer cells. Biochim Biophys Acta Mol Cell Res 1865: 1786-1794, 2018.

5. Wang QC, Zheng Q, Tan H, Zhang B, Li X, Yang Y, Yu J, Liu Y, Chai $\mathrm{H}$, Wang X, et al: TMCO1 is an ER $\mathrm{Ca}(2+)$ load-activated $\mathrm{Ca}(2+)$ channel. Cell 165: 1454-1466, 2016.

6. Michael Yates T, Ng OH, Offiah AC, Willoughby J and Berg JN; DDD Study, and Johnson DS: Cerebrofaciothoracic dysplasia: Four new patients with a recurrent TMCO1 pathogenic variant. Am J Med Genet A 179: 43-49, 2019.

7. Kondkar AA, Mousa A, Azad TA, Sultan T, Alawad A Altuwaijri S, Al-Obeidan SA and Abu-Amero KK: Polymorphism rs7555523 in transmembrane and coiled-coil domain 1 (TMCO1) is not a risk factor for primary open angle glaucoma in a Saudi cohort. J Negat Results Biomed 15: 17, 2016.

8. Li CF, Wu WR, Chan TC, Wang YH, Chen LR, Wu WJ, Yeh BW, Liang SS and Shiue YL: Transmembrane and coiled-coil domain 1 impairs the AKT signaling pathway in urinary bladder urothelial carcinoma: A characterization of a tumor suppressor Clin Cancer Res 23: 7650-7663, 2017.

9. Zhou BO, Nie J, Yang W, Huang C, Huang YE and Zhao H: Effect of hydrothorax EGFR gene mutation and EGFR-TKI targeted therapy on advanced non-small cell lung cancer patients. Oncol Lett 11: 1413-1417, 2016.

10. Pascoe HM, Knipe HC, Pascoe D and Heinze SB: The many faces of lung adenocarcinoma: A pictorial essay. J Med Imaging Radiat Oncol 62: 654-661, 2018.

11. Li Y, Yu WK, Chen L, Chan YS, Liu D, Fong CC, Xu T, Zhu G, Sun D and Yang M: Electrotaxis of tumor-initiating cells of H1975 lung adenocarcinoma cells is associated with both activation of stretch-activated cation channels (SACCs) and internal calcium release. Bioelectrochemistry 124: 80-92, 2018.

12. Déliot $\mathrm{N}$ and Constantin B: Plasma membrane calcium channels in cancer: Alterations and consequences for cell proliferation and migration. Biochim Biophys Acta 1848: 2512-2522, 2015.

13. Fan H, Shen YX and Yuan YF: Expression and Prognostic Roles of TRPV5 and TRPV6 in Non-small cell lung cancer after curative resection. Asian Pac J Cancer Prev 6: 2559-2563, 2014.

14. Ma X, Cai Y, He D, Zou C, Zhang P, Lo CY, Xu Z, Chan FL, Yu S, Chen Y, et al: Transient receptor potential channel TRPC5 is essential for P-glycoprotein induction in drug-resistant cancer cells. Proc Natl Acad Sci USA 109: 16282-16287, 2012.

15. Capiod T: Extracellular calcium has multiple targets to control cell proliferation. Adv Exp Med Biol 898: 133-156, 2016.
16. Yang LL, Liu BC, Lu XY, Yan Y, Zhai YJ, Bao Q, Doetsch PW, Deng X, Thai TL, Alli AA, et al: Inhibition of TRPC6 reduces non-small cell lung cancer cell proliferation and invasion. Oncotarget 8: 5123-5134, 2017.

17. Racioppi L, Nelson ER, Huang W, Mukherjee D, Lawrence SA, Lento W, Masci AM, Jiao Y, Park S, Liu Y, et al: CaMKK2 in myeloid cells is a key regulator of the immune-suppressive microenvironment in breast cancer. Nat Commun 10: 2450, 2019.

18. Vaz CV, Rodrigues DB, Socorro S and Maia CJ: Effect of Extracellular calcium on regucalcin expression and cell viability in neoplastic and non-neoplastic human prostate cells. Biochim Biophys Acta 1853: 2621-2628, 2015.

19. Yuan K, Chung LW, Siegal GP and Zayzafoon M: Alpha-CaMKII controls the growth of human osteosarcoma by regulating cell cycle progression. Lab Invest 87: 938-950, 2007.

20. Byun JS, Sohn JM, Leem DG, Park B, Nam JH, Shin DH, Shin JS, Kim HJ, Lee KT and Lee JY: In vitro synergistic anticancer activity of the combination of T-type calcium channel blocker and chemotherapeutic agent in A549 cells. Bioorg Med Chem Lett 26: 1073-1079, 2016.

21. He Y, Lin G, Han D, Shi D, Liu T, Gao Y, Guan W and Cheng G: Aclidinium Bromide holds promising inhibitory effects in A549 lung cancer cells potentials by regulating PI3K/AKT signaling pathway. J BUON 24: 560-565, 2019

22. Vervliet T, Parys JB and Bultynck G: Bcl-2 proteins and calcium signaling: Complexity beneath the surface. Oncogene 35: 5079-5092, 2016

23. Annunziato L, Amoroso S, Pannaccione A, Cataldi M Pignataro G, D'Alessio A, Sirabella R, Secondo A, Sibaud L and Di Renzo GF: Apoptosis induced in neuronal cells by oxidative stress: Role played by caspases and intracellular calcium ions. Toxicol Lett 139: 125-133, 2003.

24. Li G, He Y, Yao J, Huang C, Song X, Deng Y, Xie S, Ren J, Jin M and Liu H: Angelicin inhibits human lung carcinoma A549 cell growth and migration through regulating JNK and ERK pathways. Oncol Rep 36: 3504-3512, 2016.

25. Lin X, Li HR, Lin XF, Yu ME, Tu XW, Hua ZD, Lin M, Xu NL, Han LL and Chen YS: Silencing of Livin inhibits tumorigenesis and metastasis via VEGF and MMPs pathway in lung cancer. Int J Oncol 47: 657-667, 2015.

26. Shi S, Luo W, Zhang R, Wang C, Zheng Y, Song Y, Wang R, Zhang L, Zhang L, Li W and Luo Z: CRTC2 promotes non-small cell lung cancer A549 migration and invasion in vitro. Thorac Cancer 9: 136-141, 2018

27. Pastushenko I and Blanpain C: EMT Transition states during tumor progression and metastasis. Trends Cell Biol 29: 212-226, 2019.

28. Daugaard I, Sanders KJ, Idica A, Vittayarukskul K, Hamdorf M, Krog JD, Chow R, Jury D, Hansen LL, Hager H, et al: MiR-151a induces partial EMT by regulating E-cadherin in NSCLC cells. Oncogenesis 6: e366, 2017.

29. Gloushankova NA, Rubtsova $\mathrm{SN}$ and Zhitnyak IY: Cadherin-mediated cell-cell interactions in normal and cancer cells. Tissue Barriers 5: e1356900, 2017.

30. Tadokoro A, Kanaji N, Liu D, Yokomise H, Haba R, Ishii T, Takagi T, Watanabe N, Kita N, Kadowaki N and Bandoh S: Vimentin regulates invasiveness and is a poor prognostic marker in non-small cell lung cancer. Anticancer Res 36: 1545-1551, 2016.

31. Sun Z, Zhang H, Wang X, Wang QC, Zhang C, Wang JQ, Wang YH, An CQ, Yang KY, Wang Y, et al: TMCO1 is essential for ovarian follicle development by regulating ER Ca ${ }^{2+}$ Store of Granulosa cells. Cell Death Differ 9: 1686-1701, 2018

32. Alanay Y, Ergüner B, Utine E, Hacariz O, Kiper PO, Taskıran EZ, Perçin F, Uz E, Sağiroğlu MŞ, Yuksel B, et al: TMCO1 Deficiency causes autosomal recessive cerebrofaciothoracic dysplasia. Am J Med Genet A 164: 291-304, 2014. 